\title{
PSORIASIS VULGARIS AND THYROID PATHOLOGY: A POORLY KNOWN ASSOCIATION
}

\author{
Bachvarova R. \\ Clinic of Dermatology and Venereology, Medical University "Prof. Paraskev Stoyanov" - Varna \\ Reviewed by: S. Shtilionova, DM
}

\begin{abstract}
Psoriasis vulgaris is a chronic relapsing dermatosis which affects between 2 and $3 \%$ of the world population. Sometimes it causes even a durable patient's disability. Numerous recent investigations by foreign and Bulgarian authors established a correlation between psoriasis vulgaris and a series of internal diseases such as arterial hypertension, diabetes mellitus, obesity, hyperlipidemia, etc. Nowadays its definition as a systemic disease has widely been approved. The present paper reports the first clinical study carried out in Bulgaria which deals with the association between psoriasis vulgaris and thyroid diseases. The study covered 33 out-patients patients, 16 females and 17 males aged between 7 and 82 years. In 16,5\% of them, a variety of thyroid diseases was established. These alterations did not depend on patient's age. Skin manifestations did not always correlate with the results from the laboratory examinations. Skin lesions presented with single droplet-like eruptions, smaller and larger plaques of different duration as well as with inveterate plaques. Thyroid pathology presented with Hashimoto's thyroiditis, Basedow's disease, and nodules. Female gender was slightly more commonly affected. The proof of the association between psoriasis vulgaris and thyroid diseases could contribute to better control of treatment and future improvement of individual patient's quality of life.
\end{abstract}

Key words: psoriasis vulgaris, thyroid diseases, psoriasis-thyroid association, thyroid hormones, quality of life

\section{INTRODUCTION}

The patients with chronic skin diseases undergo, usually, an out-patient observation. They are more seldom hospitalized because most exacerbations are coped out of hospital. The majority of the patients pay their main attention to their dermatological status rather than to the relationship between the skin diseases and underlying internal diseases. Very few of them are aware of the fact that human skin reflects the internal environment of the organism which normal functioning determines the good external appearance and skin function as well.

The consideration of psoriasis vulgaris as a systemic disease is more widely shared nowadays $(3,5,9)$. Many recent investigations by foreign and Bulgarian authors established a correlation between psoriasis vulgaris and a series of internal diseases such as arterial hypertension, diabetes mellitus, obesity, increased risk of atherosclerosis and cardiovascular diseases, etc. (3). However, there are scanty publications about the relationship between psoriasis vulgaris and thyroid pathology. Among them, a special attention deserves the comprehensive investigation by Arican et al. (2004) of 103 psoriasis vulgaris patients. According to other published data, thyroid abnormalities present with hyperfunction, hypofunction, nodules and even carcinoma (7). Their duration varies between several months and more than 20 years. Skin manifestations con- sist not only in single and few plaques but also in large plaques. The severity of the skin disease does not always correlate with the thyroid status.

As in Bulgaria there are no studies dealing with the association between psoriasis vulgaris and thyroid pathology we decided to perform the present clinical investigation on this topic and to focus the attention of the medical community to this actual and socially significant issue.

\section{MATERIAL AND METHODS}

The present study was of cross-sectional type. It covered a total of 33 patients from the region of Varna, Bulgaria. They were 16 females and 17 males aged between 7 and 82 years. All of them were out-patients who underwent comprehensive clinical examinations during a two-month period. It stressed that along with the common accompanying diseases such as arterial hypertension, diabetes mellitus, colitis, etc., some of these patients reported a variety of conditions typical of the thyroid pathology. That was why all the dermatological out-patients were purposefully interviewed in this respect. A special attention was paid to the onset, duration, type and localization of psoriasis vulgaris in relation to the occurrence of thyroid gland disorders as well as to the presence or absence of accompanying diseases, interpreta- 
tion of the results from the laboratory examinations and patient's family history.

\section{RESULTS}

Our results showed an association of psoriasis vulgaris with thyroid diseases in $16,5 \%$ of the patients. It was established that psoriasis vulgaris can either precede the thyroid disease (in one patient), or occur in a short period (in one patient) and a longer one - of several years (in 3 patients) after it. Both young and adult individuals were affected. There was no difference concerning profession, education level, and social status. Female gender was slightly more commonly affected. There was a positive familial case history about dermal or endocrine pathology in almost all the patients. The severity of the chronic skin disease did not always correlate to the abnormalities of the laboratory examinations. Thyroid profile was severely affected in a small number of cases with slightly expressed psoriasis vulgaris, and vice versa, in other cases with severe skin disease there were only discrete aberrations of the laboratory parameters. Concerning the hormonal examinations of T4, TSH, anti-TPO and anti-TG, both TSH and anti TPO showed the most significant abnormalities from the normal values.

Psoriasis vulgaris was typically located - on elbows, knees, and trunk (in 3 patients). The only clinical manifestation consisted in dystrophic nails and erythemic-squamous plaques on head skin in two patients which represented a diagnostic problem because of their rarity. Skin lesions presented with smaller and larger plaques, sometimes of inveterate nature, droplet-like eruptions and nail alterations as well. Thyroid anomalies varied between hypofunction, hyperfunction, and adenoma. Patient's attitude towards the control of the disease varied between poor (in one patient) and good (in four patients). The duration of the disease ranged between 1-2 years and more than 40 years. Some patients shared that psoriasis vulgaris worsened in parallel to thyroid pathology. All the patients reported a poor quality of life.

\section{DISCUSSION}

Despite the advances in etiology, pathogenesis and treatment of psoriasis vulgaris, it remains an insufficiently clarified disease yet. Most probably, skin represents a target organ of thyroid hormones which contribute to the augmentation of the epidermal growth factor and thus accelerate the epidermal proliferation. Their excessive production can worsen the skin disease (7). The existence of skin T3-receptors was already proved. Recent experimental research showed they influence upon keratin synthesis. T3-receptors play a crucial role in cellular growth and differentiation. Propylthiouracil is an anti-thyroid drug used for local and peroral administration. Recent clinical studies show that it exerts a favourable therapeutic effect in the patients with psoriasis vulgaris. It is assumed that has a regulatory effect on T-cells of psoriatic plaques. Propylthiouracil increases the total amount of T-suppressor cells and reduces the activity of the lymphocytes in these plaques $(2,4)$.

Our results demonstrate that skin lesions can occur before, simultaneously, or after the endocrine disease. Usually, thyroid pathology is of chronic nature and presents with slight or atypical manifestations. That is why it is often ignored, although it undoubtedly exerts a negative effect on psoriasis vulgaris. In this respect, the diagnosis of a underlying thyroid disease can be done during certain occasional laboratory examinations. Thyroid diseases occur more often in cases with severe eruptive or long-lasting drug-resistant plaque psoriasis vulgaris. There is literature evidence that adequate treatment of the endocrine problem leads to a considerable and durable influence on the skin lesions and even to remission $(1,6,8)$. Some of our patients who strive for maintenance a good balance between these diseases have drawn the same conclusion.

\section{CONCLUSION}

Taking into consideration the insufficient clarification of the association between psoriasis vulgaris and thyroid pathology, there emerges a necessity to perform a more comprehensive and larger-scale investigation in this field. This association occurs relatively common among our patients in $16,5 \%$ of the cases. A greater number of patients should be followed-up in order to obtain more precise results enabling more reliable conclusions. The collaboration between dermatologists and endocrinologists is of essential importance, too. The elucidation of this association could contribute to better control of treatment by timely recognition of latent thyroid diseases, especially among risk contingents, by avoiding complications, ensuring remissions and reducing hospital and out-patient costs. This will result in further improvement of individual patient's quality of life.

\section{REFERENCES}

1. Arican, O., K. Bilgic, K. Koc. The effect of thyroid hormones in psoriasis vulgaris.- Indian $J$. Dermatol. Venereol. Leprol., 70, 2004, No 6, 353-356.

2. Chowdhury, M. M., R. Marks. Oral propylthiouracil for the treatment of resistant plaque psoriasis.- J. Dermatol. Treat., 12, 2001, 81-85.

3. Cohen, A. D., H. Gilutz, Y. Henkin, D. Zahger, J. Shapiro, D. Y. Bonneh, D. A. Vardy. Psoriasis and the metabolic syndrome.- Acta Derm. Venereol., 87, 2007, No 6, 506-509.

4. Elias, A. N., M. M. Goodman, W. H. Liem, J. Barr. Propylthiouracil in psoriasis: result of an open trial.- J. Am. Acad. Dermatol., 29, 1993, 78-81.

5. Humphreys, M. S., J. L. Waddell. Lithium, psoriasis, abnormal glucose tolerance, and thyroid dysfunction.- Br. J. Psychiat., 152, 1988, 437-438. 
6. Kano, Y., M. Chiba, A. Yagita, T. Shiohara. Complete resolution of psoriasis vulgaris after excision of thyroid cancer.- Int. J. Dermatol., 36, 1997, No 4, 280-282.

7. Kwinter, J., M. Weinstein, H. Bargman. Psoriasiform lesions and abscesses as initial manifestations of severe hypothyroidism in a previously healthy 15-year-old girl.- Pediatr. Dermatol., 24, 2007, No 3, 321-323.

8. Leslie, K. S., N. J. Levell. Thyroid feeding: a forgotten treatment for psoriasis.- Clin. Experim. Dermatol., 29, 2004, No 5, 567-568.

9. Yamamoto, T., A. Yokoyama. Coexistence of psoriasis vulgaris, ulcerative colitis, and thyroid adenoma.- J. Dermatol., 24, 1997, No 4, 275-276. 